\title{
END-POINT CONSTRUCTION AND SYSTEMATIC TITRATION ERROR IN LINEAR TITRATION CURVES-COMPLEXATION REACTIONS
}

\section{P. M. J. COENEGRACHT}

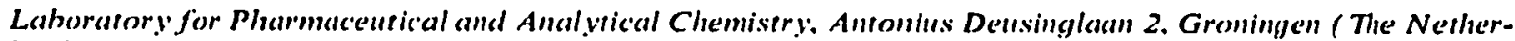
lands)

A. J. M. DUISENBERG

Lahoratorium voor Algemene Chemie. Transitorium 3, De Uithof. Utrecht (The Netherhands)

(Received 31st January 1975)

In a recent paper ${ }^{1}$ an apparatus for precise. stepwise recording of amperometric titration curves has been described. The recorded curve is atumatically corrected for dilution of the sample solution with the titrant. The end-point is found from the intersection of the extrapolated "linear" segments (ranges) on the two branches that form the titration curve. The alutomatic correction for changes in volume makes it possible to extrapolate such ranges at large distances after the end-point, where otherwise dilution effects would distort the curve. This possibility is particularly important if the titration reaction does not proceed completely: in such cases, the recorded titration curve deviates from the straight lines (Fig. 1 , curve $A$ ). and the theoretical hyperbolic form is more apparent. The curvature in the vicinity of the end-point prevents accurate extrapolation.

Careful selection of the ranges of the titration parameter, $f$, which are used for extrapolation. makes it possible to minimize the systematic titration error. Rosenthal et $a l^{2}$ have reported a few ranges which result in a small systematic titration error, but no procedure for the selection of the ranges has been given. Moreover, the length of the range used for extrapolation before the end-point always equals the length of the range used after the end-point. However, practical reasons, e.g.

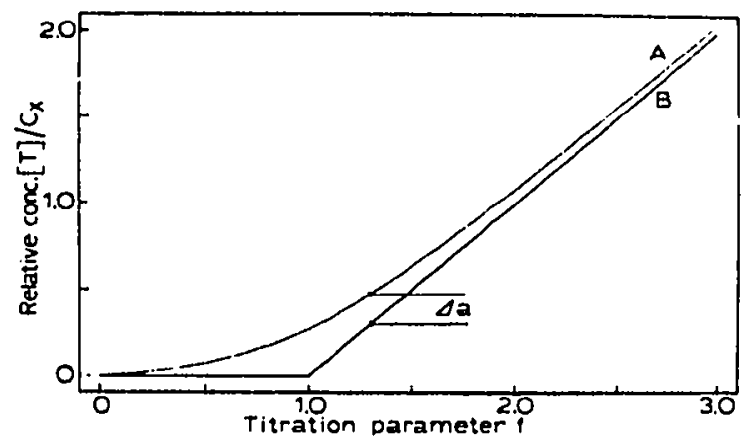

Fig. 1. Calculated normalized titration curves for the renction $X+T \neq X T$. (A) Incomplcte reaction. $K c,=10$. (B) Completc reaction, $K c_{2}=\infty$. 
a limited number of data points and partial deformation of the curve, make it desirable to be able to choose various combinations of ranges of different lengths.

In the present paper, a graphical method is proposed for the selection of these ranges, when the end-point is constructed by the intersection of tangents to the titration curve. The selection of the ranges is based on minimizing the systematic titration error. Measurement errors are not considered. First, the systematic titration error is calculated; subsequently, one can select two collections of tangents to ranges before and after the end-point, which result in a given maximum absolute titration error. As the exact selection of ranges requires that the end-point be known, ranges can only be estimated. Although the estimation has been performed with reasonable accuracy ${ }^{2}$, it remains difficult to determine the ranges on strongly bent titration curves. If the titration is continued far beyond the end-point $(f>2)$, the second branch of the curve better approximates a straight line and can be extrapolated more easily than the first branch. Thus the construction of an endpoint by the intersection of the volume axis with the tangent to the second branch of the titration curve is considered for titration curves of a reversed-L shape (Fig. 1).

\section{THEORETICAL CONSIDERATIONS}

The titration reaction of a component $X$ with a titrant $T$ to yield a soluble 1:1 product $X T$ can be represented by

$$
\mathrm{X}+\mathrm{T} \rightleftharpoons \mathrm{XT}
$$

for which the conditional formation constant is

$$
K=[\mathrm{XT}] /[\mathrm{X}][\mathrm{T}]
$$

For precipitation reactions eqn. (2) does not apply. Work on this case is in progress and leads to different results. Charges are omitted and concentrations are used instead of activities. Dilution effects can be neglected because of the automatic correction. The analytical concentrations of the component and the titrant are $c_{\mathrm{x}}$ and $c_{1}$. The titration parameter, $f$, is defined by

$$
f=c_{\mathrm{t}} / c_{\mathrm{x}}
$$

and the following equations are valid

$$
\begin{aligned}
& {[\mathrm{X}]+[\mathrm{XT}]=c_{\mathrm{x}}} \\
& {[\mathrm{T}]+[\mathrm{XT}]=c_{\mathrm{t}}=f c_{\mathrm{x}}}
\end{aligned}
$$

It is assumed that the titration curve is recorded by monitoring the concentration of only one compound involved in the reaction. The relationship between the titration parameter and the concentration of this compound is found by elimination of the other variables from eqns. (2), (4) and (5).

The relationship between $f$ and $[T]$ is represented by

$$
f=\frac{[\mathrm{T}]}{c_{\mathrm{x}}}+\frac{K[\mathrm{~T}]}{1+K[\mathrm{~T}]}
$$

and the titration curve has a reversed-L shape. Analogous expressions can be derived for the relation between $f$ and the concentration of $\mathrm{X}$ and $\mathrm{XT}^{3}$. 
Calculation of the systematic titration error

Case $A$. The end-point is found by the intersection of two tangents to the titration curve, one at a point $[\mathrm{T}]_{\mathrm{b}}, f_{\mathrm{b}}$, before the end-point, and another at a point $[\mathrm{T}]_{i, \ldots} f_{i,}$, after the end-point. The equation of a tangent to the titration curve at point $[\mathrm{T}]_{\mathrm{a}}, f_{\mathrm{a}}$, is

$$
[\mathrm{T}]=\left(\frac{\mathrm{d}[\mathrm{T}]}{\mathrm{d} f}\right)_{\mathfrak{u}}\left(f-f_{\mathfrak{a}}\right)+[\mathrm{T}]_{\mathfrak{w}}
$$

Elimination of $[T]$ from eqn. (7) and the similar equation of the tangent at point $[\mathrm{T}]_{\mathrm{b}}, f_{\mathrm{b}}$, results after rearrangement in a general expression for the titration parameter in the point of intersection $f_{i}$,

$$
f_{\mathrm{i}}=\frac{[\mathrm{T}]_{\mathrm{a}}-[\mathrm{T}]_{\mathrm{b}}+\left(\frac{\mathrm{d}[\mathrm{T}]}{\mathrm{d} f}\right)_{\mathrm{b}} f_{\mathrm{b}}-\left(\frac{\mathrm{d}[\mathrm{T}]}{\mathrm{d} f}\right)_{\mathrm{a}} f_{\mathrm{u}}}{\left(\frac{\mathrm{d}[\mathrm{T}]}{\mathrm{d} f}\right)_{\mathrm{b}}-\left(\frac{\mathrm{d}[\mathrm{T}]}{\mathrm{d} f}\right)_{\mathrm{i}}}
$$

The value of $f_{i}$ can be calculated for given values of $K c_{x}, f_{a}$ and $f_{b}$ as follows. First, all terms of the right-hand side of eqn. (8) are multiplied by $K$. When the products are calculated, eqn. (6) can be rewritten as

$$
K[\mathrm{~T}]^{2}-\left(f K c_{x}-K c_{x}-1\right)[\mathrm{T}]-f c_{x}=0
$$

Solving the quadratic equation, one obtains

$$
[\mathrm{T}]=\left\{f K c_{\mathrm{x}}-K c_{\mathrm{x}}-1+\left(\left(-f K c_{\mathrm{x}}+K c_{\mathrm{x}}+1\right)^{2}+4 f K c_{\mathrm{x}}\right)^{4}\right\} / 2 K
$$

The other root of the quadratic is physically meaningless. $K[\mathrm{~T}]_{\mathrm{u}}$ and $K[\mathrm{~T}]_{\mathrm{b}}$ are calculated from eqn. (10) for given values of $K c_{\mathbf{x}}, f_{\mathrm{a}}$ and $f_{\mathrm{b}}$. Differentiation of eqn. (6) with respect to [T] and inversion of the resulting expression yields

$$
\frac{\mathrm{d}[\mathrm{T}]}{\mathrm{d} f}=\frac{c_{\mathrm{x}}(1+K[\mathrm{~T}])^{2}}{K c_{\mathrm{x}}+(1+K[\mathrm{~T}])^{2}}
$$

For given values of $\mathrm{K} c_{\mathrm{x}}, f_{\mathfrak{a}}$ and $f_{\mathrm{h}}$, one calulates $K(\mathrm{~d}[\mathrm{~T}] / \mathrm{d} f)_{\mathrm{a}}$ and $\mathrm{K}(\mathrm{d}[\mathrm{T}] / \mathrm{d} f)_{\mathrm{b}}$ by substitution of the values of $K[T]_{\mathrm{b}}$ and $K[T]_{b}$ calculated above in eqn. (11). Subsequently $f_{i}$ is calculated by means of eqn. 8 . At the equivalence point $f_{i}=1$ and the systematic titration srror $E_{1}$ is given by

$$
E_{i}=f_{1}-1
$$

Freese $^{4}$ has shown that the systematic titration error is the same for $f-[\mathrm{T}], f-[\mathrm{X}]$ and $f-[\mathrm{XT}]$ curves.

Case $B$. The end-point is found by the intersection of the volume axis with the tangent at point $[\mathrm{T}]_{\mathrm{a}}, f_{\mathrm{a}}$, when the titration curve is of the reversed-L type. Even for low values of $K c_{x}$, the titration curve approaches a straight line at high values of the titration parameter $(f>2)$. Graphical extrapolation of this almost linear part of the curve is more convenient than extrapolation of any range before the end-point $(0 \leqslant f<1)$. Intersection with the volume axis eliminates the practically difficult extrapolation of a more or less bent part of the titration curve before the end-point. 
The relation between the point of intersection, $f_{s}$, and the ordinate of the point of contact, $[T]_{a}$, is found by substitution of $[T]=0$ in eqn. (7). From the resulting expression. $f_{\mathrm{u}}$ and $(\mathrm{d}[\mathrm{T}] / \mathrm{d} f)_{4}$ are eliminated by means of eqns. (6) and $(11)$, and after simplification the titration parameter at the point of intersection, $f_{s}$, is given by

$$
\begin{aligned}
& f_{\mathrm{s}}=\frac{K^{2}[\mathrm{~T}]_{\mathrm{a}}^{2}}{\left(K[\mathrm{~T}]_{\mathrm{i}}+1\right)^{2}} \\
& f_{4}=1+E_{\mathrm{s}}=1-\frac{2 K[\mathrm{~T}]_{\mathrm{a}}+1}{\left(K[\mathrm{~T}]_{\mathrm{a}}+1\right)^{2}}
\end{aligned}
$$

For $K[\mathrm{~T}]_{n} \gg 1$ the titration error is approximately equal to

$$
E_{4} \approx-\frac{2}{K[\mathrm{~T}]_{\text {. }}}
$$

The titration error may be expressed in terms of $K c_{x}, f_{a}$ and the fractional deviation from complete reaction. The fractional deviation (aifter the end-point) $\Delta_{n}$, is defined as the difference between the real and the "ideal" concentration of the titrant, normalized to the analytical concentration, $c_{x}$ of the component (see Fig. 1):

$$
\Delta_{\mathrm{a}}=\frac{[\mathrm{T}]_{\mathrm{a}}-\left(f_{\mathrm{a}}-1\right) c_{\mathrm{x}}}{c_{\mathrm{v}}}=[\mathrm{T}]_{\mathrm{in}} / c_{\mathrm{x}}-\left(f_{\mathrm{a}}-1\right)
$$

Combination of eqns. (14) and (15) gives:

$$
E_{*} \approx-2 /\left\{K c_{x}\left(f_{\mathrm{u}}-1+\Delta_{\mathrm{u}}\right)\right\}
$$

For increasing values of $f_{n}$, the systematic titration error decreases, but remains negative. For $f_{*}=3$, the error is approximately equal to $-1 / K c_{n}$, which is the systematic titration error when the end-point is constructed ${ }^{+}$by the intersection of the tangents at $f_{\mathrm{b}}=0$ and $f_{\mathrm{a}}=2$.

\section{RESULTS}

Case A

The end-point was constructed by the intersection of the tangent to the curve at the points $f_{\mathrm{b}}=0.0,0.1,0.2,0.3,0.4$ and 0.5 with tangents at points $f_{\mathrm{a}}$ varying from 1.0 to 4.0 . Systematic titration errors, $E_{\mathrm{i}}$, were calculated for $K c_{\mathrm{x}}=50.100$. 500 and 1000 with a programmable desk calculator (Compucorp 142E).

The results are presented in Fig. 2. Several combinations of ranges of $f_{1}$, with ranges of $f_{: 1}$ can be selected; these are determined by a given maximum absolute titration error and by the value of $K c_{v}$. The length of the range (interval) before the end-point may be increased by increments of $0.1 f$, whereas the interval of $f_{\text {; }}$ may be varied continuously.

An example will illustrate the use of the graphs. If for $K c_{x}=100$, ranges are to be found which give a maximum titration error of $\pm 1 \%$ then it can be seen from Fig. 2(a), curve B, that the intersection of the tangent at $f_{1}=0.0$ with the tangent at $f_{i 1}=2.0$ results in a titration error of $-1 \%$. The intersection of the same tangent at $f_{1}=0.0$ with a tangent at $f_{;}=4.0$ gives a titration error of $+0.3 \%$. 

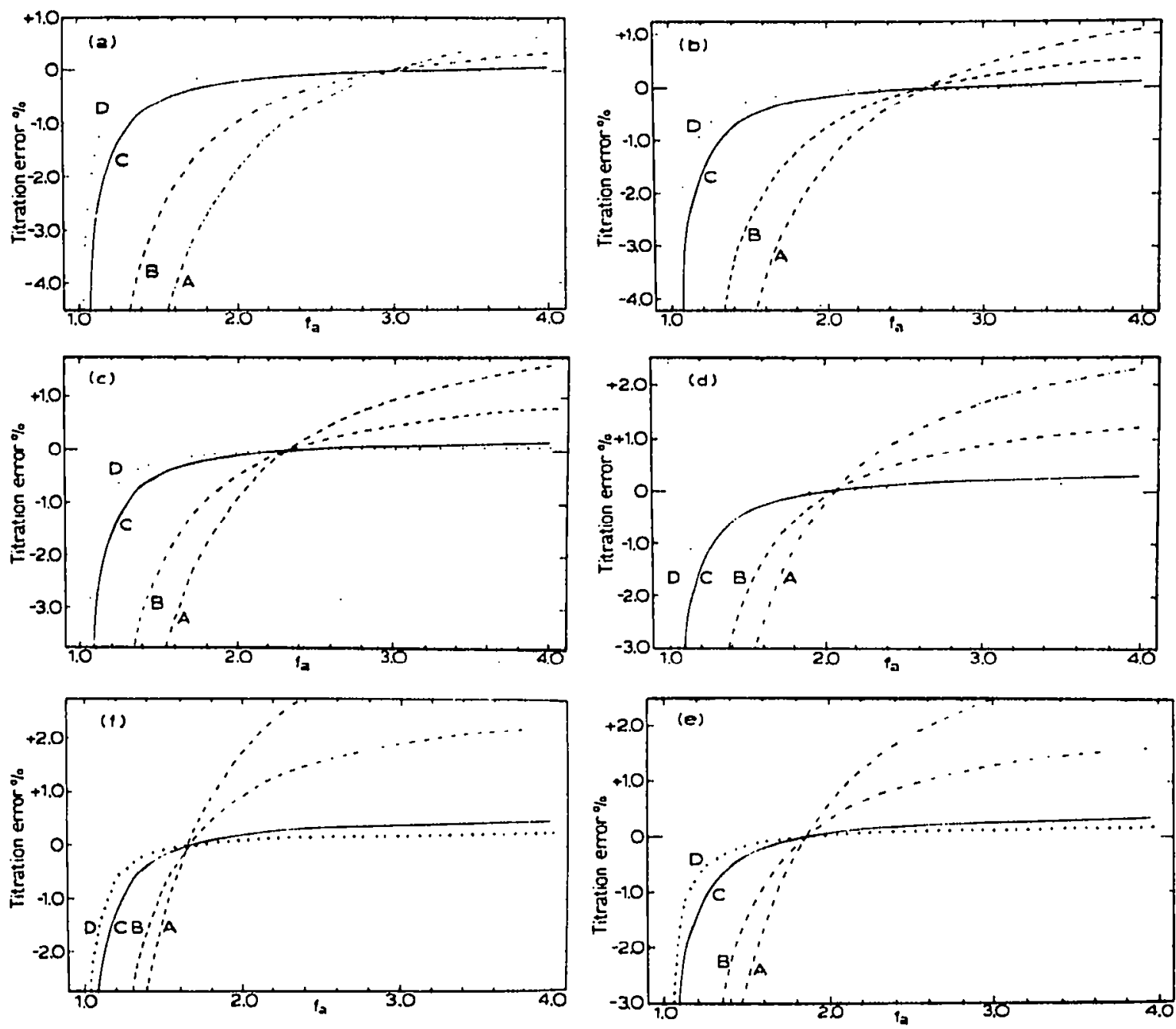

Figs. $2 a-f$. Plots of the systematic titration crror. $E_{1}$. as a function of $f_{\mathrm{a}}$ al discrete values of $f_{\mathrm{b}}$ for different values of $K c_{2}:$ (A) $K c_{k}=50 ;$ (B) $K c_{2}=100$; (C) $K c_{2}=500$; (D) $K c_{1}=1000$. Fig. 2al. $f_{h}=0.0$. Fig. 2b, $f_{h}=0.1$. Fig. 2c, $f_{h}=0.2$. Fig. 2d, $f_{h}=0.3$. Fig. 2e, $f_{h}=0.4$. Fig. 2f, $f_{h}=0.5$.

TABLE I

RANGES OF $f_{1}$ WHICH GIVE A MAXIMUM ABSOLUTE TITRATION ERROR OF $1 \%$ ON INTERSECTION BY TANGENTS AT DIFFERENT VALUES OF $f_{\mathrm{h}}$ FOR $K c_{\mathrm{r}}=100$

\begin{tabular}{lll}
\hline Fig. 2 & $f_{b}$ & $f_{\text {in runge }}$ \\
\hline $\mathrm{a}$ & 0.0 & $2.0-4.0$ \\
$\mathrm{~b}$ & 0.1 & $1.9-4.0$ \\
$\mathrm{c}$ & 0.2 & $1.8-4.0$ \\
$\mathrm{~d}$ & 0.3 & $1.7-3.4$ \\
$\mathrm{c}$ & 0.4 & $1.6-2.6$ \\
$\mathrm{f}$ & 0.5 & $1.5-2.0$ \\
\hline
\end{tabular}


It is obvious that the intersection of the tangent at $f_{\mathrm{b}}=0.0$ with any tangent at $f_{\mathrm{a}}$ between 2.0 and 4.0 gives an absolute titration error equal to or smaller than $1 \%$. This procedure can be repeated for the tangents at $f_{\mathrm{b}}=0.1,0.2,0.3,0.4$ and 0.5 (Fig. 2(b-l)). The results are given in Table $I$, and have been rounded off to the first number after the decimal point.

Ranges of $f_{\mathrm{b}}$ are chosen from the data from Table $I$ and combined with ranges of $f_{\mathrm{a}}$. Each value of $f_{\mathrm{b}}$ determines a range of $f_{\mathrm{a}}$. When a range of $f_{\mathrm{b}}$ has been chosen, then both the lower and the upper limiting value of this $f_{\mathrm{b}}$ range defines a range of $f_{u}$. The overlapping part of the two $f_{\mathrm{i}}$ ranges forms the useful range of $f_{i}$, which must be combined with the chosen range of $f_{11}$. A few examples are given : $0.0-0.2$ with $2.0-4.0 ; 0.0-0.3$ with $2.0-3.4 ; 0.1-0.4$ with $1.9-2.6$; or $0.2-0.4$ with 1.8-2.6. For the range $0.0-0.5$ no suitable range of $f_{\mathrm{a}}$ can be found. Each range is limited by two tangents. The intersection of the tangent at the beginning of the range of $f_{\mathrm{b}}$ with the tangent at the beginning of the range of $f_{\mathrm{a}}$ gives the maximum negative titration error, $-1 \%$. Likewise, the intersection of the tangents at the end of each range gives the maximum positive titration error, $+1 \%$.

In the same manner, the titration error can be represented, when the endpoint is constructed by the intersection of the tangent at discrete values of $f_{11}$ with tangents at $f_{\mathrm{b}}$ values varying from $0.0-1.0$. This is shown in Fig. $3(\mathrm{a}-\mathrm{d})$ for $f_{\mathrm{i}}=1.5$. 2.0, 2.5 and 3.0. Although it is possible to cover the whole titration curve by extension of Fig. 2(a-f) for the remaining values of $f_{b}$, Fig. 3(a-d) is given in order to show clearly the variation of the titration error with $f_{\mathrm{h}}$ for a constant value of $f_{\mathrm{u}}$. The part of the titration curve that is the most important for the construction of the end-point is covered sufficiently with Figs. 2 and 3.
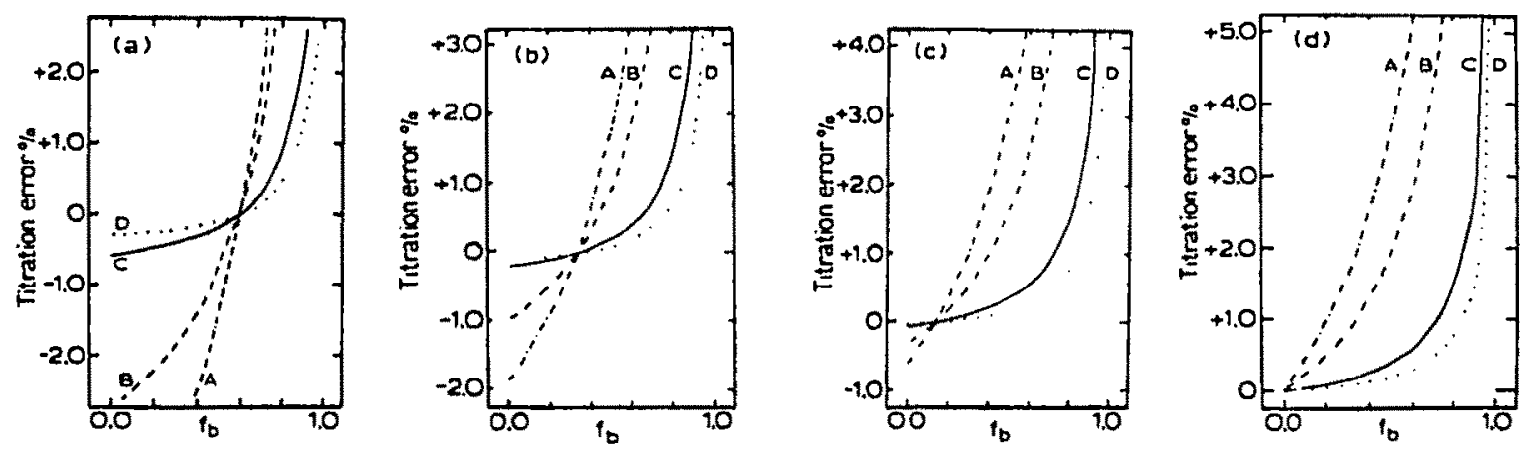

Figs. $3 a-d$. Plots of the systematic titration error, $E_{1}$, as a function of $f_{n}$ at discrete values of $f_{a}$ for different values of $K c_{x}$. (A) $K c_{x}=50$; (B) $K c_{2}=100$; (C) $K c_{2}=500$; (D) $K c_{x}=1000$. Fig. 3a, $f_{n}=1.5$. Fig. 3b, $f_{n}=2.0$. Fig. 3c. $f_{u}=2.5$. Fig. $3 \mathrm{~d}, f_{\mathrm{a}}=3.0$.

It is obvious from Fig. 3(d) that the combination of any range of $f_{\mathrm{h}}$ with a range of $f_{\mathrm{a}}$ starting at 3.0 always results in a positive titration error. Figure 4 was constructed from Figs. 2 and 3 and some additional calculations. This nomogram shows the $\pm 1 \%$ error limits for $K c_{x}=50,100,500$ and 1000 . It permits a selection of combinations of ranges that give a maximum absolute titration error of $1 \%$. First, a range of $f_{\mathrm{b}}$ can be selected by departing from the negative error limit for a given value of $K c_{x}$ at the lower limiting value of the desired $f_{b}$ range in the vertical 

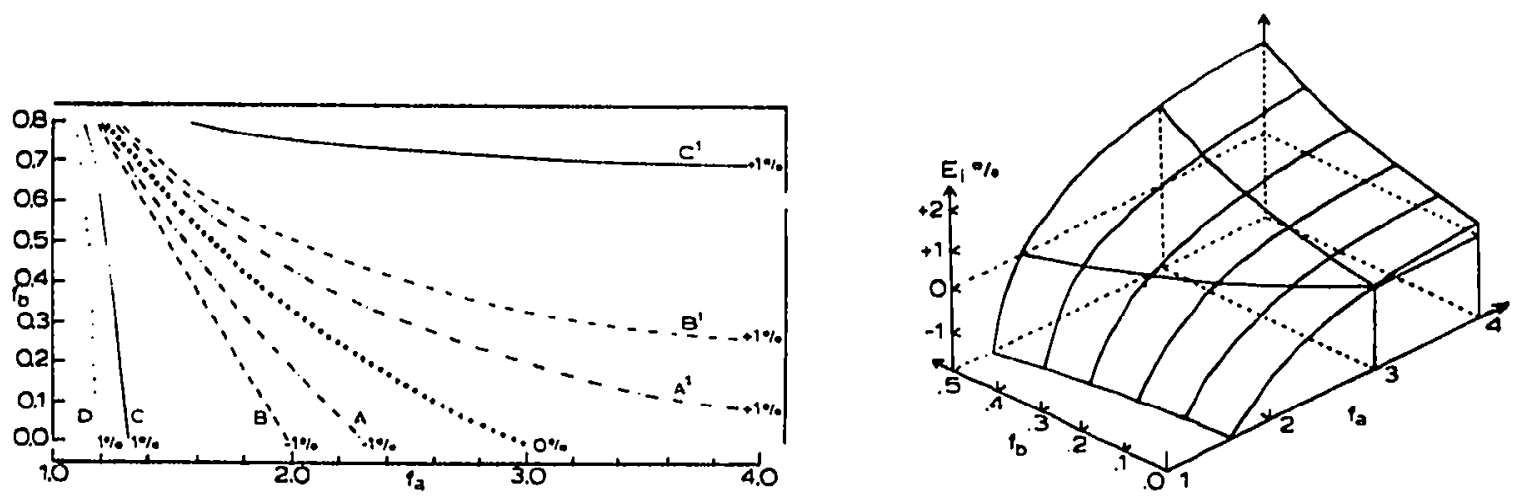

Fig. 4. Nomogram giving $\pm 1 \%$ limits of the systematic titration error, $E_{1}$. as a function of $f_{h}$ and $f_{b}$ for different values of $K c_{x}^{\prime}$. (A. A') $K c_{x}^{\prime}=50 ;\left(B, B^{\prime}\right) K c_{x}^{\prime}=100 ;\left(C . C^{\prime}\right) K c_{x}=500 ;$ (D) $K c_{x}=1000$. $D^{\prime}$ lies outside the figure. The crossed curve is the zero error limit for all values of $K C_{v}$.

Fig. 5. Model showing interrelationships between the systematic titration error. $E_{1}$, and $f_{h}$ and $f_{w}$.

direction until the desired upper limiting value of this range is reached. From this point, which also indicates the lower limiting value of the $f_{\text {a }}$ range. one goes in horizontal direction until the positive error limit for the same value of $K c_{x}$ is reached. This point indicates the upper limiting value of the range of $f_{a}$. The central crossed curve in Fig. 4 indicates the pair of tangents at $f_{\mathrm{b}}$ and $f_{\mathrm{a}}$. which intersect in $f=1$ and give zero titration error; this curve applies for every investigated value of $K c_{\mathrm{x}}$.

The titration error as a function of both points of contact $f_{h}$ and $f_{\text {is }}$ is depicted as the curved surface of Fig. 5 for $K c_{r}=100$. The curves B in Fig. $2(a-f)$ and in Fig. $3(a-d)$ are the secants of the error surface with planes perpendicular to the $f_{b}$-and the $f_{\mathrm{it}}$-axis respectively. In Fig. 4, curve B, $\mathrm{B}^{\prime}$ and the central crossed curve are secants of the error surface with planes perpendicular to the $E_{\mathrm{i}}$-axis for $E_{\mathrm{i}}=-1 \%$, $+1 \%$ and $0 \%$.

The ranges found from the Figs. are based on the intersection of tangents, whereas the end-point is actually constructed by the intersection of linearly extrapolated ranges of the titration curve. A computer program was written in order to obtain an estimate of the systematic titration error that results from the extrapolation method compared with the error from the tangent method. The program calculates a number of NP points of the normalized titration curve, i.e. [T]/c is calculated for $f$ values between 0.0 and 4.0 for $K c_{x}=50,100,500$ and 1000 by means of eqn. (10). with NP=40, 120.400. Subsequently, two ranges are chosen on the titration curve. one beginning at $f_{\mathrm{b}}=0.0$ and another at $f_{\mathrm{a}}=1.0$. Both ranges have equal lengths of $0.1 f, 0.2 f$ or $0.3 f ; f_{b}$ and $f_{b}$ are the respective central values of the ranges: $f_{b}$ and $f_{u}$ are moved along the titration curve in steps equal to half the interval $\left(0.05 f^{\circ}, 0.1 f^{\prime}\right.$ or $0.15 f$ ). The best lines through the points belonging to the ranges are calculated by a least-squares method and the systematic titration error which results from the intersection of the $f_{b}$ lines with the $f_{i l}$ lines is tabulated. These data show that the number of points has almost no inffuence on the systernatic titration error, provided that at least three points per interval are used for the calculation of the 
line. This result is not too surprising because no measurement errors and only small intervals are considered.

The ranges found by extrapolation are longer than the ranges found by the tangent method in the analogous case. For intervals of $0.1 f$ and $0.2 f$, graphs can be constructed which are almost identical with the curves of Figs. 2-4, when $f_{\mathrm{b}}$ is replaced by $f_{\mathrm{b}}$, and $f_{\mathrm{u}}$ by $f_{\mathrm{a}}$. Graphical comparison of the data for the interval of $0.3 f$ with the Figs. is not possible for all cases. but no important differences are found. and the ranges are longer than the ranges found by the tangent method. It can be concluded that the tangent method provides a cautious estimation of the desired ranges and has an inherent safety margin against a poor estimate of the end-point.

\section{Case B}

The end-point was constructed by intersection of the volume axis with tangents at $f_{\text {it }}$ values varying from 1.0 to 4.0 for a reversed-L shaped titration curve. Systematic titration errors, $E_{x}$, were calculated for $K c_{x}=50,100,500$ and 1000 . The results are presented in Fig. 6. The last term of eqn. (13(a)) was used for the calculation. The dashed line in Fig. 6 was calculated from eqn. (14). It can be seen from Fig. 6 that the titration error at $f_{\mathrm{i}}=2.0$ is twice the titration error at $f_{\mathrm{i}}=3.0$. The titration error at $f_{11}=3.0$ is indeed equal to $-1 / K c_{x}$ and it can be concluded that this construction of the end-point. which is simple to perform, results in an error no larger than the error of the conventional method, provided that the titration is continued to higher values of the titration parameter.

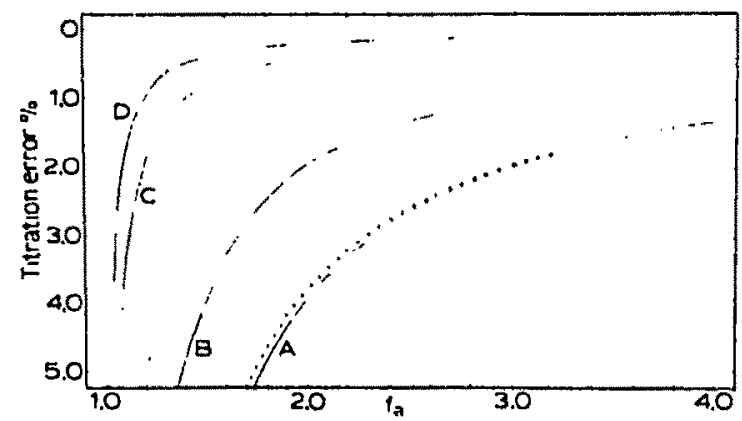

Fig. 6. Plots of the systematic titration error, $E_{x}$ as a function of $f_{\mathrm{a}}$ for diferent values of $K c_{x}$. (A) $K c_{x}=50$; (B) $K c_{x}=100 ;$ (C) $K c_{2}=500$; (D) $K c_{n}=1000$.

\section{DISCUSSION}

The exact selection of ranges yielding a given titration error for a given value of $K c_{\mathrm{x}}$ requires that the conditional formation constant and the stoichiometric end-point be known. Conditional formation constants can often be found or calculated from published data ${ }^{5.6}$, and the end-point can be estimated from the titration curve. If the conditional formation constant is unknown. one can find couples of ranges which result in small titration errors for $K c_{x}$ values of 50 (or more) from Figs. 2-4. For the selection, it is useful to have a rule of thumb, which can be 
found from Figs. 2 and 3 : the titration error is approximately inversely proportional to $K c_{\mathrm{x}}$ for titration errors smaller than about $5 \%$. Curves $\mathrm{B}$ and $\mathrm{B}^{\prime}$ in Fig. 4 can be used as the $\pm 2 \%$ error limits for $K c_{x}=50$. Now two combinations of ranges, $0.0-0.2$ with 2.3-3.0, or $0.0-0.4$ with $2.0-2.6$, can be found from the curves $A, A^{\prime}$ and $B, B^{\prime}$ of Fig. 4. which result in a titration error of $\pm 1 \%$ and of $\pm 2 \%$, resepctively, for $K c_{v}=50$. The useful ranges of $f_{11}$ usually start at $f_{1}=0.0$. If this is the case. and if the $f_{h}$ range must be smaller than about $0.4 f$ becaluse of strong curvature of the titration curve. then the $f_{i l}$ range must begin at high values of the titration parameter, usually at values greater than about $f_{i t}=2.0$. A large excess of titrant must be added. The smaller the range of $f_{\mathrm{h}}$, the higher the values of $f_{\mathrm{a}}$ which must be chosen. If the $f_{11}$ range is selected at too high values of the titration parameter, then a positive titration error is introduced. The negative titration error made by selecting the $f_{\mathrm{a}}$ range at too low values of the titration parameter is usually larger. because the negative error varies more with $f_{1}$ than the positive error. From the examples given above, it is cleitr that with Figs. 2-4. various couples of ranges can be chosen for a given titration error and for a given value of $K c$. The ranges may have different intervals before and after the end-point and the $f_{b}$, range need not begin at $f_{\mathrm{b}}=0.0$. This may be advantageous if there are experimental difficulties that cause the first measurements of the titration curve to deviate. The effort of carefully selecting ranges will be worthwhile if one wishes fully to exploit the advantages of linear methods in the titration of very dilute solutions. Then the linear segments of the titration curve are short and difficult to extrapolate.

Careful selection of ranges will not only give more accurate results. but will also help to improve precision and to shorten the time necessary for duplicate titrations. Ranges can be estimated from the first titration, which must be plotted entirely; for subsequent titrations, measurements are made only within the estimated ranges. Precision is improved by taking sumicient, i.e. 6-8, data points per range ${ }^{7.8}$. Time is gained because no measurements are done outside the ranges. Usually fewer measurements than the number needed to plot the complete titration curve will suffice. Moreover, the time per measurement can be shorter, because no measurements are made in the vicinity of the end-point, where the equilibrium is established slowly.

If the titration curve has a reversed- $L$ shape the end-point can be easily constructed by taking 6-8 measurements starting from $f_{i:}=3.0$ and by intersecting the least-squares line of the points with the volume axis. It is important that only the titrant contributes to the measured quantity. For example, in amperometric titrations the residual current must be neglible, otherwise the intersection of the leastsquares line with the volume axis will introduce a larger negative error. If the residual current is constant, the least-squares line must be intersected by the residual current line.

SUMMARY

The systematic titration error which is introduced by the intersection of tangents to hyperbolic titration curves is discussed. The effects of the apparent (conditional) formation constant, of the concentration of the unknown component and of the ranges used for the end-point construction are considered. A graphical 
method is presented for the selection of pairs of ranges which result in small systematic titration errors. The method permits the selection of pairs of ranges with equal or unequal intervals before and after the end-point. For titration curves with a reversed-L shape, the error is calculated when the end-point is constructed by the intersection of the tangent to the second branch of the curve with the volume axis. The systematic titration error is equal to $-1 / K c_{\mathrm{x}}$ when the tangent to the curve is taken at $f_{\mathrm{a}}=3.0$.

\section{REFERENCES}

1 P. M. J. Coenegracht. Anal. Chem., 45 (1973) 1675.

2 D. Rosenthal, G. L. Jones Jr. and R. Megargle, Allal. Chim. Acta, 53 (1971) 141.

3 G. den Boef, Grondslagen van de amalyse in waterige oplossingen. Agon Elsevier, Amsterdam. 1968. p. 62.

4. F. Freese, Ph.D. Thesis, Amsterdam. 1971.

5 L. G. Sillen and E. Martell, Stability' Constants of Metal Ion Complexes, The Chemical Society, London. 1964.

6 A. Ringbom. Complexation in analytical chemistr.; Interscience. New York, 1963.

7 C. Liteunu and D. Cörmös. Rev. Roum. Chim., 10 (1965) 361.

8 J. Vrestal and S. Kotrly, Talanta, 17 (1970) 151. 